\title{
Numerical simulation method for Brownian particles dispersed in incompressible fluids
}

\author{
Hiroaki Yoshida $^{\mathrm{a}, *}$, Tomoyuki Kinjo ${ }^{\mathrm{b}}$, Hitoshi Washizu ${ }^{\mathrm{b}, \mathrm{c}, 1}$ \\ ${ }^{a}$ Toyota Central RED Labs., Inc., Bunkyo-ku, Tokyo 112-0004, Japan \\ ${ }^{b}$ Toyota Central ReD Labs., Inc., Nagakute, Aichi 480-1192, Japan \\ ${ }^{c}$ Elements Strategy Initiative for Catalysts and Batteries (ESICB), Kyoto University, Kyoto 615-8245, Japan
}

\begin{abstract}
We present a numerical scheme for simulating the dynamics of Brownian particles suspended in a fluid. The motion of the particles is tracked by the Langevin equation, whereas the host fluid flow is analyzed by using the lattice Boltzmann method. The friction force between a particle and the fluid is evaluated correctly based on the velocity difference at the position of the particle. The coupling method accurately reproduces the long-time tail observed in the velocity auto-correlation function. We also show that the fluctuation-dissipation relation holds between the relaxation of a single particle and the velocity autocorrelation function of fluctuating particles.
\end{abstract}

Keywords: Colloidal suspensions, Brownian particles, Langevin dynamics, Lattice Boltzmann method

\section{Introduction}

Colloidal suspensions of sub-micro or nano particles play important roles in many situations. Examples include processes of producing secondary-battery electrodes and coats of paint [1, 2]. Recently, functional fluids that change their rheological properties have also attracted attentions [3, 4]. The particles suspended in solutions in those systems thermally fluctuate, which is referred to as the Brownian motion. In order to understand the connection between this microscopic motion of the particles and the macroscopic fluid properties, the hydrodynamic motion of the solution induced by the Brownian particles has to be comprehended correctly.

The computational method that is most widely used for simulating the Brownian motion of particles is the one solely based on the Langevin equation, referred to as the Brownian dynamics simulation. The simplest approach in implementation of the Brownian dynamics simulation assumes that the solvent is at rest and not affected by the motion of the particles, where random force representing the thermal fluctuation and an effective friction force in proportion to the particle velocity exert on a particle. To include the hydrodynamic interaction effect into the fluctuation and friction forces is still challenging despite a number of attempts that have been made to incorporate the effect of the motion of the surrounding fluid [5--13], because simulating directly the motion of the fluid is required to capture the hydrodynamic interaction effect that stays within the fluid at the time scale of the momentum dissipation. There exist two types of simulation methods in which the fluid flow is directly

\footnotetext{
${ }^{*}$ Corresponding author.

Email address: h-yoshida@mosk.tytlabs.co.jp (Hiroaki Yoshida)

${ }^{1}$ Present address: Graduate School of Simulation Studies, University of Hyogo, 7-1-28 Minatojima-minamimachi, Chuo-ku, Kobe, Hyogo 650-0047,
} Japan. simulated along with tracking the motion of the particles: (i) a particle has finite size comparable with the computational domain for the fluid flow, and a boundary condition at the surface of the particle is imposed in the flow simulation [14-19], (ii) a particle is represented by a single point, and a model friction force is employed in order to incorporate the interaction between the particle and the fluid [20-23]. The latter is advantageous from the point of view of computational cost, but the inaccuracy of the local estimation of the friction force can be a problem in certain physical situations.

In this work, we present an accurate local estimation of the fluid-particle friction force. Specifically, the friction force acting on a particle is estimated by fitting the analytical solution for the flow around a Stokes-let to the flow field obtained numerically. The reaction force acts on the position of the particle, which realizes two-way coupling between the particle and fluid motions. The lattice Boltzmann method is employed for the flow simulation, which is compatible with massive parallel computing, and is easy to apply various types of boundary conditions such as the periodical shear boundary, and complex structure of obstacles. For validation of the method, the long-time tail observed in the velocity auto-correlation function (VACF) is compared with the analytical expression. The fluctuation-dissipation theorem, which relates the VACF and the relaxation process of the velocity and acceleration of a single particle, is also examined.

\section{Numerical algorithm}

\subsection{Estimation of the friction force}

We first describe the equation governing the motion of Brownian particles, and present the algorithm for coupling with the fluid flow. The motion of the particles is described by the 
Langevin equation in the following form:

$$
\begin{aligned}
& M_{i} \frac{\mathrm{d} \boldsymbol{V}_{i}}{\mathrm{~d} t}=\boldsymbol{F}_{C i}+\boldsymbol{F}_{H i}+\boldsymbol{F}_{R i}, \\
& \boldsymbol{V}_{i}=\frac{\mathrm{d} \boldsymbol{r}_{i}}{\mathrm{~d} t},
\end{aligned}
$$

where $M$ is the mass, $\boldsymbol{r}_{i}$ is the position, and $\boldsymbol{V}_{i}$ is the velocity, of $i$ th particle. In Eq. (1), the conservative inter-particle force, the dissipation force, and the fluctuation force are denoted by $\boldsymbol{F}_{C}$, $\boldsymbol{F}_{H}$, and $\boldsymbol{F}_{R}$, respectively. In the present study, we employ the conservative force derived from the potential of conventional dissipative particle dynamics [24] having the following form:

$$
U\left(\bar{r}_{i j}\right)=a_{f}\left(\bar{r}_{i j}-\frac{1}{2} \bar{r}_{i j}^{2}\right),
$$

where $a_{f}$ is a coefficient determining the intensity of the interparticle force, and $\bar{r}_{i j}$ is the inter-particle distance. If the particle density is not significantly large, the choice of $\boldsymbol{F}_{C}$ has limited effect on the results, because the hydrodynamic interaction through the fluid flow acts as a repulsive force. The fluctuation force meets the following property:

$$
\begin{aligned}
& \left\langle F_{R i}^{p}\left(t, \boldsymbol{r}_{i}\right)\right\rangle=0, \\
& \left\langle F_{R i}^{p}\left(t, \boldsymbol{r}_{i}\right) F_{R i}^{q}\left(t^{\prime}, \boldsymbol{r}_{i}^{\prime}\right)\right\rangle=\sigma^{2} \delta(p-q) \delta\left(t-t^{\prime}\right) \delta\left(\boldsymbol{r}_{i}-\boldsymbol{r}_{i}^{\prime}\right),
\end{aligned}
$$

where $\langle\cdot\rangle$ denotes the ensemble average, $\delta$ is the Kronecker delta, and $\sigma$ is a constant determining the intensity of the random force. Here, superscripts $p$ and $q$ indicate the Cartesian components of the force.

In the simplest Brownian dynamics simulation, the dissipation force is proportional to the velocity of the particle itself $\boldsymbol{V}(t)$ :

$$
\boldsymbol{F}_{H}=-\gamma \boldsymbol{V}(t),
$$

where $\gamma$ is the constant for the friction force related to the fluid viscosity via Stokes' law: $\gamma=6 \pi \eta R$ with $R$ representing the particle radius. Since Eq. (6) assumes the fluid to be at rest, the momentum transport through the fluid is neglected, and thus the Brownian motion of particles at the time scale of the momentum dissipation is not described accurately. For example, the VACF $C_{v}(t)=\langle\boldsymbol{V}(0) \cdot \boldsymbol{V}(t)\rangle$, which should exhibit the so-called long-time tail described by a power function of time $\left(C_{v}(t) \propto t^{-3 / 2}\right)$, decays exponentially if we use Eq. (6) $\left(C_{v}(t) \propto \exp (-\gamma t / M)\right)$, as will be discussed later in Sec. 3 . In order to capture the hydrodynamic interaction effect, Dünweg and his co-workers proposed the friction force to be in proportion to the particle velocity relative to the fluid velocity at the position of the particle, which is obtained from an independent simulation of the Navier-Stokes equations: [20, 21]

$$
\boldsymbol{F}_{H i}=-\gamma\left(\boldsymbol{V}_{i}(t)-\boldsymbol{u}\left(t, \boldsymbol{r}_{i}\right)\right)
$$

where $\boldsymbol{u}$ is the velocity field of the fluid. This correction certainly realizes a momentum transport through the fluid, and the long-time tail is qualitatively reproduced. However, the underestimation of the friction force due to the local estimation results in an insufficient accuracy in reproducing the VACF, as they recognized, and the fluctuation-dissipation theorem is thus not satisfied without an empirical tuning of the coefficient $\gamma$.

In the present study, we replace the flow velocity $\boldsymbol{u}$ in Eq. (7), i.e., the flow velocity of the fluid at the particle position, by the flow velocity away from the position of the particle $\boldsymbol{u}^{\infty}$, still estimated locally. Since the flow velocity away from the particle is not unique, we use the value of $\boldsymbol{u}^{\infty}$ obtained assuming that the flow velocity field around the particle is approximated by the one-way flow field around a spherical particle. Since, in the actual numerical simulation, the flow velocity is obtained at a regular computational grid by using the lattice Boltzmann method, the value of $\boldsymbol{u}^{\infty}$ is evaluated using the flow velocity at the neighboring grid points $\boldsymbol{u}_{p}^{\mathrm{N}}=\boldsymbol{u}\left(\boldsymbol{x}_{p}\right)$, where $\boldsymbol{x}_{p}(p=1, \ldots, 8)$ represents the neighboring eight grid points around the particle.

To this end, we use the following analytical solution of the flow past a Stokes-let with the intensity $6 \pi \eta R U^{\infty}$ :

$$
\boldsymbol{u}^{\mathrm{A}}=U^{\infty}\left[\left(1-\frac{3}{4} \frac{R}{r}\right) \boldsymbol{e}_{x}-\frac{3 R x}{4 r^{3}} \boldsymbol{x}\right] .
$$

Here, the flow in the $x$-direction is assumed, and the Stokes-let is at the origin ( $\boldsymbol{e}_{x}$ is a unit vector in the $x$-direction, and $\boldsymbol{x}$ is the spatial coordinate,). After transforming Eq. (8) such that the origin is at the particle position, and the flow is in the direction of the vector $\boldsymbol{u}_{p}^{\mathrm{N}}-\boldsymbol{V}$ averaged over eight points (unit vector in this direction is denoted by $\boldsymbol{e}^{\mathrm{N}}$ ), we calculate the difference between the relative flow velocity $\boldsymbol{u}_{p}^{\mathrm{N}}-\boldsymbol{V}$ at grid points and the $\boldsymbol{u}^{\mathrm{A}}$ (Eq. (8)) at corresponding points. The value of $U^{\infty}$ is then determined to minimize the sum of the squares of the difference, $\sum_{p=1}^{8}\left|\boldsymbol{u}_{p}^{\mathrm{N}}-\boldsymbol{V}-\boldsymbol{u}^{\mathrm{A}}\left(\boldsymbol{x}_{p}\right)\right|^{2}$. Using the value of $U^{\infty}$ determined, the flow velocity away from the particle is obtained as $\boldsymbol{u}^{\infty}=$ $U^{\infty} \boldsymbol{e}^{\mathrm{N}}+\boldsymbol{V}$.

In the flow simulation by means of the lattice Boltzmann method, the momentum due to the motion of the particle is transferred via the reaction force of $\boldsymbol{F}_{H}$ :

$$
\boldsymbol{F}_{p i}=-\boldsymbol{F}_{H i} \delta\left(\boldsymbol{x}-\boldsymbol{r}_{i}\right) / \Delta V,
$$

where $\Delta V$ is the unit cell volume of the computational grid system. The pointwise force is distributed around the neighboring grid points with a weight depending on the distance from $\boldsymbol{r}_{i}$.

\subsection{Outline of the lattice Boltzmann method}

In the present study, we employ the lattice Boltzmann method (LBM) to obtain the flow velocity $\boldsymbol{v}(t, \boldsymbol{x})$ and the pressure $p(t, \boldsymbol{x})$ governed by the Navier-Stokes equations:

$$
\begin{aligned}
& \nabla \cdot \boldsymbol{v}=0, \\
& \frac{\partial \boldsymbol{v}}{\partial t}+(\boldsymbol{v} \cdot \nabla) \boldsymbol{v}=-\frac{1}{\rho_{0}} \nabla p+v \nabla^{2} \boldsymbol{v}+\frac{1}{\rho_{0}} \sum_{i} \boldsymbol{F}_{p i},
\end{aligned}
$$

where $\rho_{0}$ is the reference fluid density and $v$ is the kinetic viscosity.

In the LBM, the flow behavior is described in terms of the velocity distribution function $f_{\alpha}(t, \boldsymbol{x})$, instead of directly handling the variables $\boldsymbol{v}$ and $p$. Here, $\alpha=0,1,2, \ldots, n$ with $n$ being the number of discrete velocities. The value of $f_{\alpha}$ represents the partial fluid density, each of which travels over the 
regular lattice with the discrete velocity assigned. The direction of the discrete velocity is defined in terms of the vector $\boldsymbol{e}_{\alpha}$. There are several sets of the discrete velocities, which satisfy the constraints that must be satisfied in order to reproduce the Navier-Stokes equations. We here employ the fifteen-velocity set, one of the most widely used sets (see, e.g., Ref. [25] for the specific expression). The relation between the local density of the fluid and the velocity distribution function is:

$$
\tilde{\rho}=\sum_{\alpha} f_{\alpha}
$$

where $\tilde{\rho}$ is the density normalized by $\rho_{0}$. Since the incompressible fluid is considered, $\tilde{\rho}$ should be close to unity, and thus the value of $f_{\alpha}$ is regarded as a discrete probability distribution function. Therefore, the velocity of the fluid is expressed as:

$$
\boldsymbol{u}=\sum_{\alpha} C \boldsymbol{e}_{\alpha} f_{\alpha}
$$

where $C$ is the speed defined in terms of the time step $\Delta t$ and the grid interval $\Delta x$ as $C=\Delta x / \Delta t$. The basic equation of the LBM then reads

$$
f_{\alpha}\left(t+\Delta t, \boldsymbol{x}+\boldsymbol{e}_{\alpha} \Delta x\right)=f_{\alpha}(t, \boldsymbol{x})+Q_{\alpha}[f](t, \boldsymbol{x})+\Delta t \omega_{\alpha} G_{\alpha},
$$

where $Q_{\alpha}$ is the collision operator that defines interaction between $f_{\alpha}$ 's:

$$
Q_{\alpha}[f]=\frac{1}{\tau}\left[f_{\alpha}^{\mathrm{eq}}(\tilde{\rho}(t, \boldsymbol{x}), \boldsymbol{u}(t, \boldsymbol{x}))-f_{\alpha}(t, \boldsymbol{x})\right] .
$$

Here, $\tau$ is the relaxation-time coefficient in relation with the fluid viscosity:

$$
v=\frac{1}{3}\left(\tau-\frac{1}{2}\right) \frac{\Delta x^{2}}{\Delta t},
$$

and $f_{\alpha}^{\text {eq }}$ is the equilibrium distribution function defined as

$$
f_{\alpha}^{\mathrm{eq}}(\tilde{\rho}, \boldsymbol{u})=\omega_{\alpha}\left[\tilde{\rho}+\frac{3}{C} u_{j} e_{j \alpha}+\frac{9}{2 C^{2}}\left(u_{j} e_{j \alpha}\right)^{2}-\frac{3}{2 C^{2}} u_{j}^{2}\right],
$$

where $\omega_{\alpha}$ is a weight coefficient, of which the specific expression is dependent on the set of discrete velocities (cf. Table 5.1 of Ref. [25]). The interaction force $\boldsymbol{F}_{p i}$ acts on the fluid through $G_{\alpha}$ defined as

$$
G_{\alpha}=\frac{3 F_{p j} e_{\alpha j}}{\rho_{0} C} .
$$

We summarize the computational process of the LBM. Given the distribution function at $t, f_{\alpha}(t+\Delta t, \boldsymbol{x})$ is obtained through two steps, namely,

(i) Collision process:

$$
\hat{f}_{\alpha}(t, \boldsymbol{x})=f_{\alpha}(t, \boldsymbol{x})+Q_{\alpha}[f](t, \boldsymbol{x})+\Delta t \omega_{\alpha} G_{\alpha} .
$$

(ii) Streaming process:

$$
f_{\alpha}\left(t+\Delta t, \boldsymbol{x}+\boldsymbol{e}_{\alpha} \Delta x\right)=\hat{f}_{\alpha}(t, \boldsymbol{x}) .
$$

Then, we calculate the physical quantities $\tilde{\rho}$ and $\boldsymbol{u}$ using Eqs. (12) and (13). The pressure $p$ is expressed in terms of

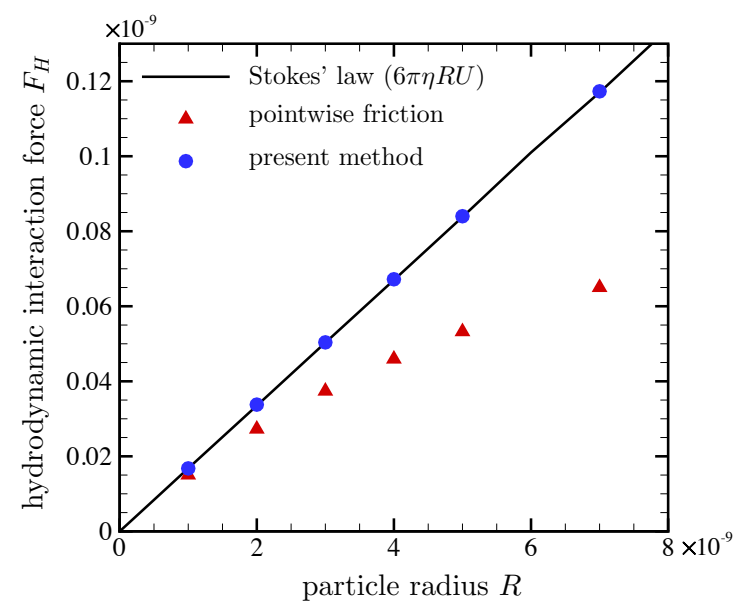

Figure 1: Hydrodynamic interaction force $F_{H}$ acting on a sphere at rest in a uniform flow $U$. The symbol $\boldsymbol{\Delta}$ indicates the results obtained using the pointwise friction model (7), whereas the symbol $\bullet$ indicates the results of the present method. The solid line is Stokes's formula $F_{H}=6 \pi \eta R U$, where $\eta=\rho_{0} v$. $U=1[\mathrm{~m} / \mathrm{s}], v=0.89 \times 10^{-6}\left[\mathrm{~m}^{2} / \mathrm{s}\right]$, and $\rho_{0}=10^{3}\left[\mathrm{~kg} / \mathrm{m}^{3}\right]$. The size of the simulation box is $500 \times 10^{-9}[\mathrm{~m}]$, the grid interval for the lattice Boltzmann method is $\Delta x=5 \times 10^{-9}$, and the time step is $\Delta t=0.5 \times 10^{-12}[\mathrm{~s}]$.

the local density $\tilde{\rho}$ as $p=C^{2} \rho_{0} \tilde{\rho} / 3$. It is proven that the above process yields an approximated solution to the incompressible Navier-Stokes equation [26-28].

To conclude this section, we remark a few points on the parameter ranges and extensions. Firstly, since the relative velocity $U^{\infty}$ between the flow and a particle is computed using the neighboring grid points around the particle, it should not cover all these neighboring grid points i.e., we should ensure that $R \lesssim \sqrt{3} \Delta x$. Otherwise the possible modification is to use the other grid points than the nearest points in estimation of $U^{\infty}$, say eight corners of $(2 \Delta x)^{3}$ cube. The second remark is on the density of the particle suspension. For the same reason for the estimation of $U^{\infty}$, the spacing between particles should not always be small, requiring the density not being too large; we have checked the present method works correctly for the volume fraction below 0.1 . Finally we remark on the possibility of including particle rotations. Since the flow field around a rotating sphere decays more quickly than the one considered herein, the approximation of the present method should be sufficient in most cases to capture the basic properties of suspensions. Nevertheless, this extension would be possible with estimating the rotational friction using a Stokes flow solution around a rotating sphere, with the similar technique for Eq. (8).

\section{Numerical results}

The existing model given in Eq. (7) fails to accurately reproduce the friction force acting on a spherical particle on which the stick boundary condition is imposed, as mentioned in Sec. 2.1. In order to confirm the improvement of the present method, we here consider a very simple problem of a flow past a single spherical particle that is fixed $(\boldsymbol{V}(t)=0)$. Figure 1 plots the force acting on the particle as a function of the particle radius. When the Reynolds number is defined as $\operatorname{Re}=U R / v$, 


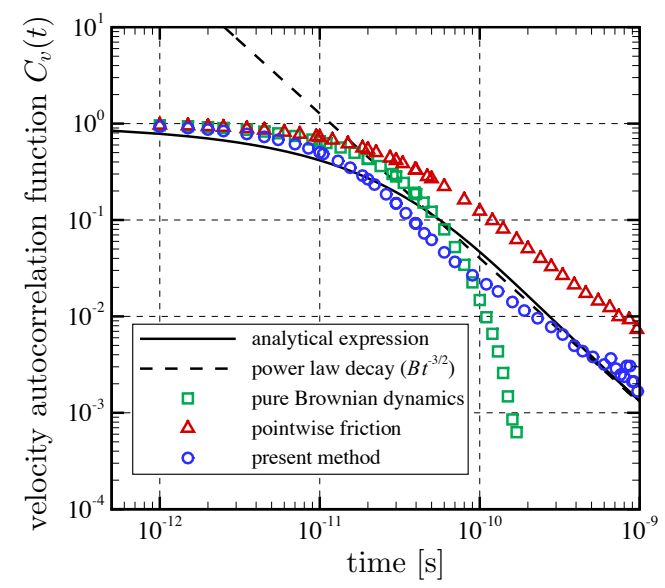

Figure 2: Velocity autocorrelation function $C_{v}(t)$ normalized by $C_{v}(0)$ for fluctuating Brownian particles. The suspension consists of 500 identical particles with $R=5 \times 10^{-9}[\mathrm{~m}]$ and $M=2 \times 10^{-21}[\mathrm{~kg}]$. Other conditions are the same as Fig. 1] The symbol $\square$ indicates the result of the pure Brownian dynamics (Eq. 6) , the symbol $\triangle$ indicates the result obtained using the pointwise friction model (Eq. (7)), and the symbol $\mathrm{O}$ indicates the result of the present method. The solid line is the analytical expression for $C_{v}(t)$ [29, 31, and the dashed line is its long-time limit $\left(C_{v}(t) / C_{v}(0) \sim B t^{-3 / 2}\right.$ with $\left.B=M /\left[12 \rho_{f}(\pi v)^{3 / 2}\right]\right)$.

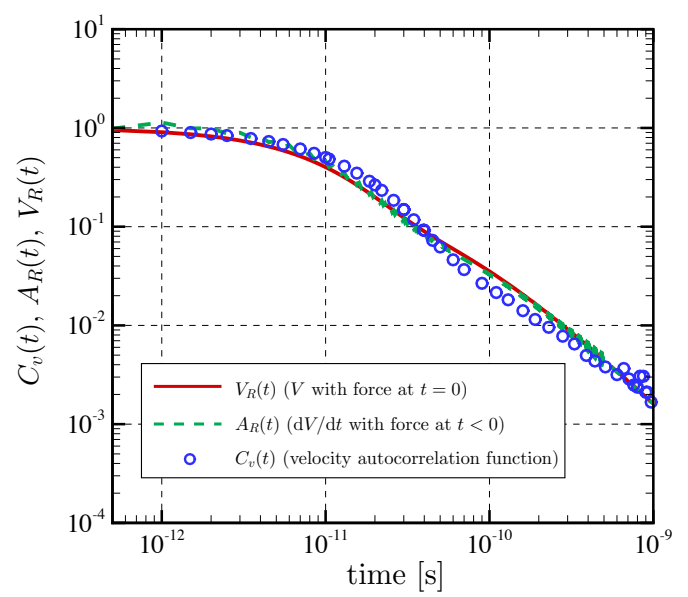

Figure 3: Velocity autocorrelation function $C_{v}(t)(\mathrm{O})$ compared to response function $V_{R}(t)$ (solid line) and relaxation function $A_{R}(t)$ (dashed line).

with $U$ being the given flow velocity at infinity, is small, the friction force is a linear function of the radius, $F_{H}=6 \pi \eta R U$ (Stokes' law). Since the relative velocity $|\boldsymbol{u}(t, \boldsymbol{r})|$ estimated at the position of the particle is obviously smaller than $U$, the friction is underestimated by Eq. (7). On the contrary, the present method estimates locally, with the aid of the flow velocity $\boldsymbol{u}_{p}^{\mathrm{N}}$ near the particle, a value of $\boldsymbol{u}^{\infty}$ close to $U$, hence the model evaluates correctly the friction force which agrees well with Stokes' law.

We next consider a system with dispersed particles in thermal equilibrium, to examine the VACF $C_{v}(t)$ of the particles. Whereas the usual Brownian dynamics with the friction force given by Eq. (6) yields a $C_{v}(t)$ decaying exponentially with respect to $t$, the memory effect due to the interaction with the surrounding fluid makes $C_{v}(t)$ decay more slowly, showing the long-time tail. The generalized Langevin equation [32] supplemented with the friction force at a small Reynolds number gives an analytical expression of $C_{v}(t)$, which shows $C_{v}(t) / C_{v}(0) \sim$ $B t^{-3 / 2}$ as $t \rightarrow \infty$, where $B=M /\left[12 \rho_{f}(\pi v)^{3 / 2}\right.$ ] [29-31]. In Fig. 2. along with the analytical expression, we plot the VACF $C_{v}(t)$ obtained using Eq. (6), Eq. (7), and the present method using $\boldsymbol{u}^{\infty}$. Although the result of Eq. (7) reproduces the long-time tail, the value of $C_{v}(t)$ still differs form the analytical expression. On the other hand, the VACF obtained using the present method exhibits better quantitative agreement with the analytical expression. There is a slight discrepancy of the present method in the region around $t=10^{-10}[\mathrm{~s}]$. This is a numerical error inherent to the LBM due to a small pressure wave which originates from the artificial compressibility.

As a consequence of the fluctuation-dissipation theorem, the relaxation processes of the velocity and acceleration coincide with the VACF. More precisely, if we denote with $V_{R}(t)$ the history of the velocity $V(t)$ of a particle kicked by an instant force at $t=0$, and we denote with $A_{R}(t)$ the acceleration $\mathrm{d} V / \mathrm{d} t(t)$ of a particle after a force exerted on the particle in $-\infty<t<0$ is released. Then the relation $C_{V}(t)=V_{R}(t)=-A_{R}(t)$ holds (both $V_{R}$ and $A_{R}$ are normalized by the values at $t=0$ ). The relation is known as Onsager's regression hypothesis that was derived from the fluctuation-dissipation theorem by Callen and Welton [33]. In Fig. 3, we plot $V_{R}(t), A_{R}(t)$ and $C_{v}(t)$ obtained using the present method for the hydrodynamic interaction. Fairly good agreement among the three functions confirms that the thermal equilibrium state of particles in a solvent is correctly simulated using the present coupling method.

\section{Conclusion}

In the present study, a numerical algorithm for simulating the behavior of a fluid with Brownian particles is presented, in which the motion of the particles is tracked by means of the Langevin equation, while the fluid flow obeying the NavierStokes equations is simulated using the LBM. The relative velocity between the particle and the fluid is evaluated from the local information, which improves the accuracy of the hydrodynamic interaction force. Several numerical simulations are performed to check the following features: (1) the friction force acting on a pinned particle in a one-way flow satisfies Stokes' law. (2) the long-time behavior of the VACF predicted theoretically is reproduced correctly. (3) the relaxation process under given disturbances agrees with the VACF (Onsager's regression hypothesis). The proposed algorithm yields correct hydrodynamic interaction force when the particles are not close, or the number density is not very large. The possibility of extending the present method to highly dense suspensions is now under investigation.

\section{Acknowledgments}

The authors are grateful to S. Iwai for computer assistance in preparing the manuscript. Part of the work was supported by MEXT program "Elements Strategy Initiative to Form Core 
Research Center" (since 2012). (MEXT stands for Ministry of Education, Culture, Sports, Science, and Technology, Japan.)

\section{References}

[1] F. Ma, Y. Fu, V. Battaglia, R. Prasher, Microrheological modeling of lithium ion battery anode slurry, J. Power Sources 438 (2019) 226994.

[2] C. Agbo, W. Jakpa, B. Sarkodie, A. Boakye, S. Fu, A review on the mechanism of pigment dispersion, J. Disper. Sci. Technol. 39 (6) (2018) 874889.

[3] H. Khodadadi, S. Aghakhani, H. Majd, R. Kalbasi, S. Wongwises, M. Afrand, A comprehensive review on rheological behavior of mono and hybrid nanofluids: effective parameters and predictive correlations, Int. J. Heat Mass Transf. 127 (2018) 997-1012.

[4] S. Arietaleaniz, P. Malgaretti, I. Pagonabarraga, R. Hidalgo, Rheological behavior of colloidal suspension with long-range interactions, Phys. Rev. E 98 (4) (2018) 042603.

[5] D. L. Ermak, J. A. McCammon, Brownian dynamics with hydrodynamic interactions, J. Chem. Phys. 69 (1978) 1352.

[6] J. F. Brady, G. Bossis, Stokesian dynamics, Ann. Rev. Fluid Mech. 20 (1988) 111-157.

[7] D. Nie, J. Lin, A fluctuating lattice-Boltzmann model for direct numerical simulation of particle Brownian motion, Particuology 7 (6) (2009) 501506.

[8] R. R. Schmidt, J. G. H. Cifre, J. G. de la Torre, Comparison of Brownian dynamics algorithms with hydrodynamic interaction, J. Chem. Phys. 135 (2011) 084116.

[9] M. Maxey, Simulation methods for particulate flows and concentrated suspensions, Annu. Rev. Fluid Mech. 49 (2017) 171-193.

[10] C. Rettinger, U. Rüde, A coupled lattice Boltzmann method and discrete element method for discrete particle simulations of particulate flows, Comput. Fluids 172 (2018) 706-719.

[11] D. Papavassiliou, N. Pham, O. Kadri, R. Voronov, Lattice Boltzmann methods for bioengineering applications, in: Numerical Methods and Advanced Simulation in Biomechanics and Biological Processes, Elsevier, 2018, pp. 415-429.

[12] Z. Liu, Y. Zhu, J. R. Clausen, J. B. Lechman, R. R. Rao, C. K. Aidun, Multiscale method based on coupled lattice-Boltzmann and Langevindynamics for direct simulation of nanoscale particle/polymer suspensions in complex flows, Int. J. Numer. Meth. Fluids (published online 2019).

[13] A. M. Lattanzi, X. Yin, C. M. Hrenya, A hybrid lattice Boltzmannrandom walk method for heat transfer in gas-solids systems, J. Comput. Phys. X 1 (2019) 100007.

[14] Y. Nakayama, R. Yamamoto, Simulation method to resolve hydrodynamic interactions in colloidal dispersions, Phys. Rev. E 71 (2005) 036707.

[15] T. Iwashita, R. Yamamoto, Short-time motion of Brownian particles in a shear flow, Phys. Rev. E 79 (2009) 031401.

[16] A. J. C. Ladd, R. Verberg, Lattice-Boltzmann simulations of particle-fluid suspensions, J. Stat. Phys. 104 (5) (2001) 1191-1251.

[17] J. Onishi, A. Kawasaki, Y. Chen, H. Ohashi, Lattice Boltzmann simulation of capillary interactions among colloidal particles, Comput. Math. Appl. 55 (2008) 1541-1553.

[18] R. G. M. van der Sman, MRT lattice Boltzmann schemes for confined suspension flows, Comput. Phys. Commun. 181 (2010) 1562-1569.

[19] L. Mountrakis, E. Lorenz, A. Hoekstra, Revisiting the use of the immersed-boundary lattice-Boltzmann method for simulations of suspended particles, Phys. Rev. E 96 (1) (2017) 013302.

[20] P. Ahlrichs, B. Dünweg, Lattice-Boltzmann simulation of polymersolvent systems, Int. J. Mod. Phys. C 9 (8) (1998) 1429-1438.

[21] P. Ahlrichs, B. Dünweg, Simulation of a single polymer chain in solution by combining lattice Boltzmann and molecular dynamics, J. Chem. Phys. 111 (1999) 8225-8239.

[22] V. Lobaskin, B. Dünweg, A new model for simulating colloidal dynamics, New J. Phys. 6 (1) (2004) 54.

[23] A. Chatterji, J. Horbach, Combining molecular dynamics with lattice Boltzmann: A hybrid method for the simulation of (charged) colloidal systems, J. Chem. Phys. 122 (2005) 184903.

[24] R. D. Groot, P. B. Warren, Dissipative particle dynamics: Bridging the gap between atomistic and mesoscopic simulation, J. Chem. Phys. 107 (1997) 4423-4435.
[25] S. Succi, The lattice Boltzmann equation for fluid dynamics and beyond, Oxford Univ. Press, New York, 2001.

[26] M. Junk, A. Klar, L.-S. Luo, Asymptotic analysis of the lattice Boltzmann equation, J. Comput. Phys. 210 (2005) 676-704.

[27] M. Junk, Z. Yang, Asymptotic analysis of lattice Boltzmann boundary conditions, J. Stat. Phys. 121 (2005) 3-35.

[28] H. Yoshida, H. Hayashi, Transmission-reflection coefficient in the lattice Boltzmann method, J. Stat. Phys. 155 (2014) 277-299.

[29] E. J. Hinch, Application of the Langevin equation to fluid suspensions, J. Fluid Mech. 72 (1975) 499-511.

[30] G. L. Paul, P. N. Pusey, Observation of a long-time tail in Brownian motion, J. Phys. A 14 (12) (1981) 3301

[31] J. T. Padding, A. A. Louis, Hydrodynamic interactions and Brownian forces in colloidal suspensions: Coarse-graining over time and length scales, Phys. Rev. E 74 (3) (2006) 031402.

[32] R. Kubo, The fluctuation-dissipation theorem, Rep. Prog. Phys. 29 (1) (1966) 255.

[33] H. B. Callen, T. A. Welton, Irreversibility and generalized noise, Phys. Rev. 83 (1951) 34-40. 